\title{
Burden of COPD in Nepal
}

This article was published in the following Dove Press journal: International Journal of COPD

\section{Tara Ballav Adhikari' \\ Dinesh Neupane ${ }^{1,2}$ \\ Per Kallestrup ${ }^{3}$}

'Nepal Development Society, Chitwan, Nepal; ${ }^{2}$ Global Health Research

Center, Duke Kunshan University, Kunshan, People's Republic of China; ${ }^{3}$ Center for Global Health (GloHAU), Department of Public Health, Aarhus University, Aarhus, Denmark

Correspondence: Tara Ballav Adhikari Nepal Development Society,

Bharatpur 10, Chitwan, Nepal Email adhikaritaraballav@gmail.com
Background: COPD is a globally significant public health problem and is the second leading cause of mortality. This study presents the health burden of COPD in Nepal using the Global Burden of Disease (GBD) study 2016 dataset.

Methods: This study used the data from the GBD repository presenting morbidity and mortality attributed to COPD, by sex and age. In GBD 2016, due to a lack of the primary source of data in Nepal, estimations on morbidity and mortality of COPD were based on its predictive covariates. Years of life lost (YLLs) were calculated based on the cause of death estimations, applying GBD's Cause of Death Ensemble modeling. Likewise, years lived with disability (YLDs) were calculated by multiplying the prevalence of each sequela by the disability weight. Disability-adjusted life years (DALYs) were derived as the sum of YLLs and YLDs.

Results: Between 1990 and 2016, the estimated age-standardized mortality rate due to COPD was decreasing for both genders, but the decline was much higher among males. Unlike the high rate of incidence among males, the age-standardized DALYs were found to be high among females $(2,274.9$ [95\% UI: 1,702.0-2,881.5] per 100,000). YLLs contributed around $80 \%$ of DALYs due to COPD in 2016. Age-standardized YLLs rate was higher among females, with a value of 1,860 (95\% uncertainty interval (UI): 1,282.8-2,472.8) vs 1,547.6 (95\% UI: $992.1-$ $2,018.5$ ) among the males per 100,000 population.

Conclusion: The prevalence and incidence of COPD remained almost stationary over the years, but still very high. Though the incidence and prevalence of disease were high among males, the death rate and DALYs were more significant among females throughout the years. If the current situation prevails, the burden of COPD will continue to increase in the country. Hence, comprehensive social, environmental, and behavioral approaches to curtail the risk factors along with early identification, treatment, and management of COPD is of utmost importance.

Keywords: DALYs, disability, mortality, burden of illness, GBD

\section{Introduction}

COPD is a disease characterized by a degeneration of lung function over time that is not entirely reversible, and it encompasses both emphysema and chronic bronchitis. ${ }^{1}$ It is a significant public health problem globally, being the second leading cause of mortality. ${ }^{2}$ As of 2016, the Global Burden of Disease (GBD) Study estimated that about 3 million people worldwide died of COPD. ${ }^{3}$ Also, COPD exerts a high economic burden on the individual as well as the health care system as it incurs two- to four-fold higher costs compared with asthma and ischemic heart disease. ${ }^{4,5}$

The World Health Organization (WHO) reports that 90\% of COPD-accounted deaths occur in low- and middle-income countries. Furthermore, it has been projected that COPD-associated mortality will increase by $160 \%$ in the Southeast Asian region in the coming decades. ${ }^{6}$ Globally, cigarette smoking, increase in longevity of populations, 
and air pollution are regarded as major risk factors for COPD. ${ }^{4,7}$ Exposure to such risk factors is even higher in lowand middle-income countries like Nepal. Some subnational studies conducted in Nepal have reported the prevalence of COPD ranging from $23 \%$ to $43 \%{ }^{8-10}$ A systematic analysis of GBD 2015 ranked Nepal as one of the top four countries with highest age-standardized disability-adjusted life years (DALYs) rates due to COPD in 2015 along with Papua New Guinea, India, and Lesotho. ${ }^{11}$ COPD is the most (43\%) common noncommunicable disease (NCD) among outpatients followed by cardiovascular disease. ${ }^{8}$ With a high level of indoor and outdoor pollution ${ }^{12}$ and consistently high number of smokers, ${ }^{13}$ COPD and its related morbidity and mortality is increasing. ${ }^{14}$

The Government of Nepal's commitment toward the Sustainable Development Goals and the National Health Policy 2014 are the recent policy directives to focus on the prevention and control of NCDs in the country. ${ }^{15}$ Similarly, the Government of Nepal, in alignment with the Global commitment for $25 \%$ relative reduction in premature mortality from NCDs by $2025,{ }^{16}$ has drafted the Multisectoral Action Plan for the Prevention and Control of NCDs (20142020). Along with this the Ministry of Health is currently working to restructure health governance, draft a new health policy and design province based health care delivery system for the new Federal Structure of the country. Understanding the national prevalence, morbidity, and mortality associated with major NCDs with higher public health and socioeconomic impact, like COPD, is essential for evidence-informed policy planning and program implementation. The study aims to present the health burden of COPD in Nepal using the GBD 2016 dataset.

\section{Methods}

This study used the publicly available data from the WHO and the Institute of Health Metrics and Evaluation GBD repository. This paper presents the trend in prevalence and incidence of COPD in Nepal and the trends of mortality and DALYs attributed to COPD in the period 1990-2016 by sex and age. The study methodology and data collection procedures of the GBD 2016 study have been comprehensively reported previously. ${ }^{17,18}$

In the GBD 2016 study, the data on causes of COPD and attributed deaths were derived from a larger database of vital registration, surveys, published and unpublished papers, and verbal debriefed data. Then, the data were analyzed using GBD's Cause of Death Ensemble modeling tool. Likewise, COPD-caused nonfatal estimations in GBD 2016 were based on systematic reviews of published papers, unpublished reports, and survey data available in GBD's Global Health Data Exchange repository. But due to the lack of a primary data source from Nepal, the estimation of morbidity and mortality was based on predictive covariates of COPD, namely, cumulative cigarette smoking for 5/10/20 years, smoking prevalence, second-hand smoke, indoor and outdoor air pollution, use of biomass for cooking or heating, and occupational exposures, and with the data from the South Asian region. ${ }^{11}$ The different surveys and research papers published during the past 27 years have been considered as the data source for estimation of COPD burden. All the papers and publications are available in GBD 2016 Data Input Sources Tool for Nepal. ${ }^{19}$ The years of life lost (YLLs) calculation was made by multiplying the number of deaths due to COPD by the remaining life expectancy in GBD's standard life table. ${ }^{20}$ Likewise, years lived with disability (YLDs) was calculated by multiplying the prevalence by the disability weight. Finally, DALYs was derived as the totality of YLLs and YLDs. ${ }^{17}$

\section{Ethical statement}

This study used deidentified publicly available data from the Global Burden of Disease Study 2016 repository. Thus, ethical approval and an ethical statement from an institutional review board or ethics committee was not required for secondary analysis of data for Nepal.

\section{Results}

In 2016, an estimated 16,302 people (95\% UI: 12,941.419,290.7) died from COPD in Nepal. Between 1990 and 2016, the mortality rate due to COPD was decreasing for both genders, but the decline was much higher among males when compared with females. Thus, by 2016, the age-standardized death rate due to COPD for the females was 119.7 per 100,000 people (95\% UI: $85.4-154.4)$, while for the males it was 102.6 per 100,000 people (95\% UI: 69.8-131.7) (Figure 1).

Likewise, 960,737 Nepalese suffered from COPD in 2016, which is twice the number of sufferers in 1990. The age-standardized prevalence rate of COPD has remained almost stagnant (4,899.1 per 100,000 population in $1990 \mathrm{vs}$ $4,810.3$ per 100,000 in 2016) over 26 years, with continued higher prevalence among Nepalese males than females (Figure 2). During the same period, the age-standardized incidence rate did not change significantly (398.5 per 100,000 population in 1990 to 382.1 per 100,000 population 


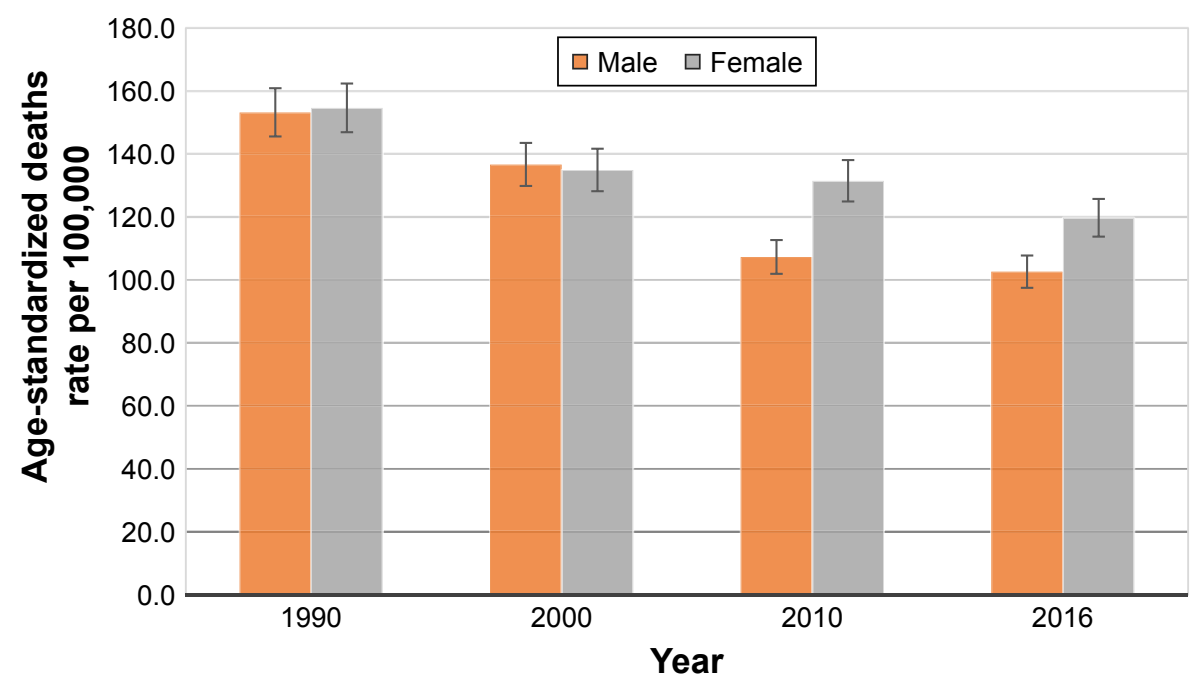

Figure I Age-standardized death rates of COPD in Nepal during 1990-2016, by sex.

in 2016) (Figure 2). However, the incidence continued to remain higher among males than females throughout the period. In 2016, the age-standardized incidence rate of COPD for males was $30.8 \%$ higher than that for Nepalese females.

Similar to death rate, morbidity caused by COPD was declining from 1990 to 2016. Unlike high rate of incidence among males in Nepal, the age-standardized DALYs was found to be high among female. In 2016, the age-standardized DALYs was 2,274.9 (95\% UI: 1,702.0-2,881.5) per 100,000 population and 2,013.3 (95\% UI: 1,457.8-2,484) per 100,000 population for females and males, respectively (Table 1). YLLs contributed around $80 \%$ of DALYs due to COPD in 2016 , which is less than its almost $85 \%$ contribution in 1990. Similarly, premature loss of life was higher among females, with age-standardized YLLs of 1,860 (95\% UI:
1,282.8-2,472.8) per 100,000 population, while for the males the YLLs rate was 1,547.6 (95\% UI: 992.1-2,018.5) per 100,000 population (Table 1). Unlike YLLs, the agestandardized YLDs was high among Nepalese males throughout the 26 years period when compared with the female counterparts.

Not surprisingly, with the increase in the age (group) of the population the overall prevalence rate, death rate and DALYs rate were found to increase steadily (Figure 3).

\section{Discussion}

The data for the GBD study on COPD for Nepal between 1990 and 2016 indicated a high and almost stagnant agestandardized prevalence and incidence of COPD. During the same period, the decrease in COPD mortality and morbidity rate was significantly large. This estimates reflects that

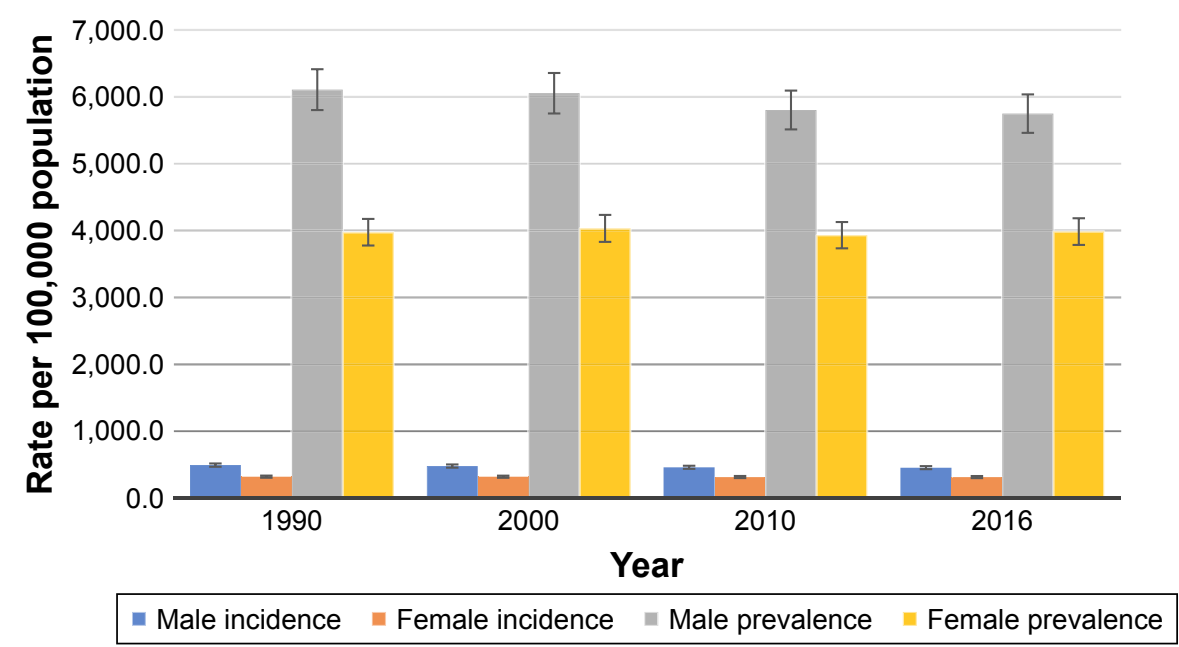

Figure 2 Age-standardized incidence and prevalence rate of COPD during 1990-2016 in Nepal, by sex. 
Table I Burden of COPD in Nepal (age-standardized rate, per 100,000 population)

\begin{tabular}{|c|c|c|c|c|c|c|c|c|c|}
\hline \multirow[t]{2}{*}{ Year } & \multicolumn{3}{|c|}{ DALYs } & \multicolumn{3}{|l|}{ YLDs } & \multicolumn{3}{|l|}{ YLLs } \\
\hline & Total & Male & Female & Total & Male & Female & Total & Male & Female \\
\hline 1990 & $3,091.7$ & $3,060.9$ & $3,121.2$ & 464.0 & 512.3 & 428.1 & $2,627.7$ & $2,548.6$ & $2,693.1$ \\
\hline 2000 & $2,680.1$ & $2,702.2$ & $2,669.3$ & 463.6 & 506.2 & 430.6 & $2,216.6$ & $2,195.9$ & $2,238.7$ \\
\hline 2010 & $2,315.3$ & $2,124.1$ & $2,499.9$ & 438.6 & 470.4 & 410.5 & I,876.7 & $\mathrm{I}, 653.7$ & $2,089.4$ \\
\hline 2016 & $2,149.4$ & $2,013.3$ & $2,274.9$ & 438.2 & 465.7 & 414.9 & I,7||.2 & I,547.6 & I,860.0 \\
\hline
\end{tabular}

Note: Global Burden of Disease Study 2016. Available from: http://vizhub.healthdata.org/gbd-compare.

Abbreviations: DALYs, disability-adjusted life years; YLDs, years lived with disability; YLLs, years of life lost.

greater improvements have been achieved so far in reducing case fatality and management of disease rather than in comprehensive health promotion approach to reducing incidence and prevalence of COPD. Various sociobehavioral and environmental factors such as smoking, use of biomass fuels, use of motorized vehicles, and other sources of outdoor air pollution play a significant role in degrading air quality in Nepal. According to the Nepal Demographic and Health Survey 2016, 27\% of the Nepalese males and 6\% of females smoked tobacco, $66 \%$ of households used solid fuel for cooking, and $31 \%$ of households were exposed to second-hand smoke, a slight decrease in indicators from the Nepal Demographic and Health Survey 2011. 21,22 However, the use of the motorized vehicle has risen from 244,000 in $1990 / 2000$ to 1.3 million in 2012/2013, which is one of the major contributors of outdoor air quality in the urban areas the country. ${ }^{23}$ With rapid urbanization, high emission of PM2.5 $\left(>40 \mu \mathrm{g} / \mathrm{m}^{3}\right)$, lack of regulation, and ineffective implementation to control the air quality, ambient air pollution has created a conducive environment for COPD ${ }^{23}$ Thus, this interplay between decreasing behavioral and indoor air pollution factors but increasing ambient air pollution may have led to consistently high yet stagnant prevalence and incidence of disease over the decade. The current prevalence of COPD ( 4,810 per 100,000 population [ $95 \%$ UI: $4,591.2-$ $5,021.6])$ is higher than the global rate $(3,628$ per 100,000 population [95\% UI: 3,486.3-3,765.75]) but lower than South Asia's regional average (5,315.9 per 100,000 population [95\% UI: 5,097.9-5,532.3]). As the Nepalese population is aging, the burden of COPD is likely to increase because with increasing age the incidence as well as death and DALYs rate were found to be increased tremendously.

The Nepalese demography is continuously aging. Seven percent of the current population is above 60 years of age, while it was $6 \%$ in 2011 and only $4 \%$ in 2006. Similarly, the life expectancy of Nepalese population has increased

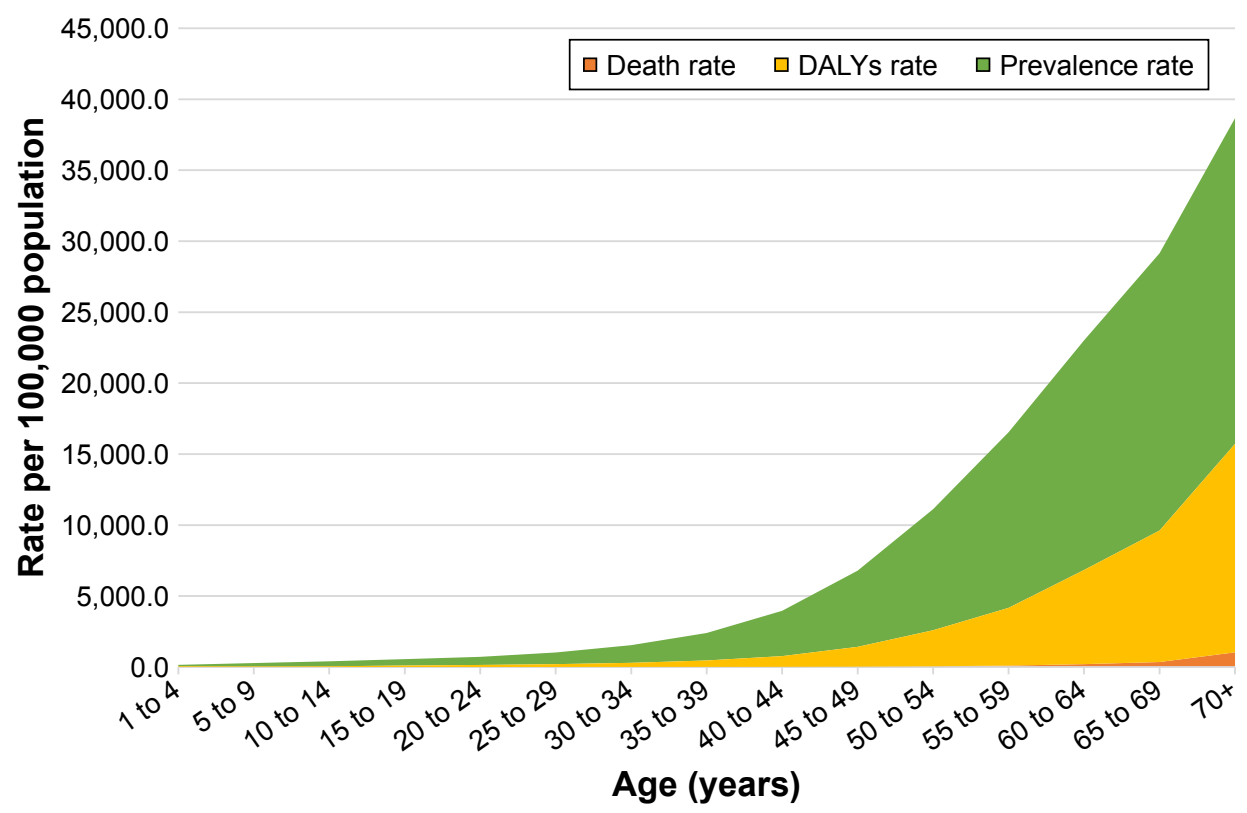

Figure 3 Age-wise COPD prevalence, mortality, and DALYs in Nepal in 2016. Abbreviation: DALYs, disability-adjusted life years. 
from 54 years in 1990 to 70 years in $2015 .^{24}$ As more people are living longer lives, the risk of occurrence of COPD and associated DALYs and mortality will also increase. Contrary to this, the age-standardized prevalence and incidence and death and disability rate in 2016 were lower than that of 1990 despite the higher proportion of the aging population. One of the reasons behind this could be advancing health care technology for COPD which leads to early detection and treatment of COPD, thus preventing premature death and disability. But if the current situation continues to remain same and the proportion of aging population increase significantly, it is likely that burden of COPD will increase extravagantly.

One of the interesting findings of the study was the high age-standardized prevalence and incidence of COPD among Nepalese males while the age-standardized death rate and DALYs were comparatively high among females. This is in contrast to the global and regional burden of COPD where the age-standardized prevalence, incidence, mortality, and morbidity rate were more significant among males as compared to females for the year 2016. Currently, Nepal is among the leading countries in COPD-related DALYs and mortality, second only to India in South Asia, whereas the mortality rate caused by COPD among females is even higher than that of India. ${ }^{25}$

The fact that more Nepalese females have either lost their life or lived with disability due to COPD despite the lower prevalence and incidence than males can be linked to the significant role played by household air pollution in development and progression of COPD. About two-thirds of households in Nepal use firewood as a source of fuel for cooking. ${ }^{26}$ Firewood used in traditional cooking stoves with poorly ventilated homes lead to indoor air pollution, mainly affecting females and children who, as per social norms, spend more time in the kitchen for household chores as compared to their male counterparts. ${ }^{27}$ One of the studies conducted among households using traditional stoves in Southern Nepal showed that PM2.5 level $\left(1,376 \mu \mathrm{g} / \mathrm{m}^{3}\right)$ was 40 times higher and carbon monoxide concentration of $10.9 \mathrm{ppm}$ was $30 \%$ higher than recommended by WHO guidelines. ${ }^{28}$ The higher degree of vulnerability for females being exposed to indoor air pollution and leading to severe forms of COPD have been reported by other studies conducted in Nepal. ${ }^{29}$

Likewise, Noncommunicable Disease Risk Factors: WHO STEPwise approach to Surveillance (STEPS) survey, Nepal 2013 reported the smoking behavior among males as being $27 \%$ and $10.3 \%$ in females. ${ }^{30}$ The prevalence of females smoking cigarettes in Nepal is much higher than the world average of $6.2 \%$, while it is almost similar for the case of males (males: $31.1 \%$ and females: $6.2 \%$ ). ${ }^{31}$

Another aspect related to higher premature loss of life due to COPD among females can be linked with the poor health-seeking behavior. Studies conducted in Nepal and other middle-income countries show that the health of the female is often ignored due to gender disparity; also, females tend to have longer delays in seeking formal health care services. ${ }^{32}$ Thus, there is higher likelihood that females visit health facilities only when the disease has reached severe stages and hence have higher disability rate.

Some evidence suggests that female suffering from COPD is associated with a reduction of lung function even with low smoking exposure, suggesting higher susceptibility to smoking effects than males. ${ }^{33}$ However, further studies investigating potential biological differences and associated COPD severity need to be performed before making concrete conclusions.

The present study has some limitations seen for the GBD estimation in case of Nepal. Due to the lack of primary data on COPD-related morbidity and mortality, GBD 2016 estimates were based on predictive covariates, namely, smoking, second-hand smoke, air pollution, biomass for cooking or heating, and occupational exposures. ${ }^{11}$ So, the actual morbidity and mortality figure may differ from the presented figure. Other newly established individual risk factors of COPD, such as low level of physical activity, ${ }^{34}$ are not considered as predictive covariates in this GBD estimation; these could also have contributed to the unexplained COPD burden in Nepal. ${ }^{35}$ Nonetheless, the estimates and publications of GBD align with Guidelines for Accurate and Transparent Health Estimates Reporting: The GATHER Statement, which defines best practices for documenting studies that synthesize evidence from multiple sources to quantitatively describe past and current population health and its determinants. ${ }^{36}$

However, in a resource-constrained setting like Nepal where reliable health statistics on COPD are very limited, these findings from the GBD study provide a reliable base for comparison of national, subregional, and global estimates for COPD and provide a framework for setting public health priorities and creating evidence-informed policies regarding COPD.

\section{Conclusion}

From 1990 to 2016, COPD continued to remain one of the major public health problems in Nepal. The age-standardized prevalence and incidence of COPD remained almost stagnant 
over the years, but still very high, while there was a significant declining trend of age-standardized mortality rate and DALYs rate with regard to COPD over the same period. Though the incidence and prevalence of disease was high among males, the death rate and DALYs rate were significantly higher among females throughout the years. If the current situation prevails, it is expected that the burden of COPD will continue to increase over the years. Hence, comprehensive social, environmental, and behavioral approaches to curtail the risk factors, ie, decreasing the prevalence of smoking behavior, replacing the use of biomass fuels, and reduction of outdoor air pollution, along with early identification, treatment, and management of COPD, is of utmost importance.

\section{Data availability}

The study is based on publicly available data from Global Burden of Disease Study 2016. The data can be downloaded from http://ghdx.healthdata.org/gbd-results-tool.

\section{Acknowledgments}

The authors are grateful to the Global Burden of Disease Study 2016 team for making the data available. We thank Kate Muller from Institute of Health Metrics and Evaluation, University of Washington for her support.

\section{Author contributions}

TBA and DN contributed to the conception of the work. TBA wrote the first draft of the manuscript with significant inputs from DN and PK. All authors contributed toward data analysis, all drafts and critically revising the paper and agree to be accountable for all aspects of the work.

\section{Disclosure}

The authors report no conflicts of interest in this work.

\section{References}

1. Viegi G, Pistelli F, Sherrill D, Maio S, Baldacci S, Carrozzi L. Definition, epidemiology and natural history of COPD. Eur Respir J. 2007;30(5):993-1013.

2. López-Campos JL, Tan W, Soriano JB. Global burden of COPD. Respirology. 2016;21(1):14-23.

3. Adeloye D, Chua S, Lee C, et al. Global and regional estimates of COPD prevalence: Systematic review and meta-analysis. J Glob Health. 2015;5(2):020415.

4. Chapman K, Mannino D, Soriano J, et al. Epidemiology and costs of chronic obstructive pulmonary disease. Eur Respir J. 2006;27(1):188-207.

5. Halbert R, Natoli J, Gano A, Badamgarav E, Buist AS, Mannino D. Global burden of COPD: systematic review and meta-analysis. Eur Respir J. 2006;28(3):523-532.

6. World Health Organization. WHO Report on the Global Tobacco Epidemic, 2008: The MPOWER Package. Geneva: World Health Organization; 2008.

7. Milavetz G. Global Surveillance, Prevention and Control of Chronic Respiratory Diseases: A Comprehensive Approach. Thousand Oaks, CA: SAGE Publications; 2008.
8. Bhandari GP, Angdembe MR, Dhimal M, Neupane S, Bhusal C. State of non-communicable diseases in Nepal. BMC Public Health. 2014; 14(1):23.

9. Pokharel B, Humagain S, Pant P, Gurung R, Koju R, Bedi T. Spectrum of diseases in a medical ward of a teaching hospital in a developing country. J Coll Med Sci Nepal. 2012;8(2):7-11.

10. Bhandari R, Sharma R. Epidemiology of chronic obstructive pulmonary disease: a descriptive study in the mid-western region of Nepal. Int $J$ Chron Obstruct Pulmon Dis. 2012;7:253.

11. Soriano JB, Abajobir AA, Abate KH, et al. Global, regional, and national deaths, prevalence, disability-adjusted life years, and years lived with disability for chronic obstructive pulmonary disease and asthma, 1990-2015: a systematic analysis for the Global Burden of Disease Study 2015. Lancet Respir Med. 2017;5(9):691-706.

12. Kim BM, Park JS, Kim SW, et al. Source apportionment of PM 10 mass and particulate carbon in the Kathmandu Valley, Nepal. Atmos Environ. 2015;123:190-199.

13. Sreeramareddy CT, Ramakrishnareddy N, Harsha Kumar HN, Sathian B, Arokiasamy JT. Prevalence, distribution and correlates of tobacco smoking and chewing in Nepal: a secondary data analysis of Nepal Demographic and Health Survey-2006. Subst Abuse Treat Prev Policy. 2011;6(33):1-9.

14. Mishra SR, Neupane D, Bhandari PM, Khanal V, Kallestrup P. Burgeoning burden of non-communicable diseases in Nepal: a scoping review. Global Health. 2015;11(1):32.

15. Gautam R. NCDs in Nepal: burgeoning burden amid low priority and the ways forward. Health Prospect. 2013;11:iv-v.

16. World Health Organization. Global Action Plan for the Prevention and Control of Noncommunicable Diseases 2013-2020. Geneva: World Health Organization; 2013.

17. Hay S. Global, regional, and national incidence, prevalence, and years lived with disability for 328 diseases and injuries for 195 countries, 1990-2016: a systematic analysis for the Global Burden of Disease Study 2016. Lancet. 2017;390(10100):1211-1259.

18. Steel N. Global, regional, and national age-sex specific mortality for 264 causes of death, 1980-2016: a systematic analysis for the Global Burden of Disease Study 2016. Lancet. 2017;390(10100):1151-1210.

19. Institute for Health Metrics and Evaluation (IHME). Global Burden of Disease Study 2016 (GBD 2016) Data Input Sources Tool; 2017. Available from: http://ghdx.healthdata.org/gbd-2016/data-input-sources? locations $=164 \&$ components $=1$. Accessed on November 20, 2017.

20. Abajobir AA, Abate KH, Abbafati C, et al. Global, regional, and national disability-adjusted life-years (DALYs) for 333 diseases and injuries and healthy life expectancy (HALE) for 195 countries and territories, 1990-2016: a systematic analysis for the Global Burden of Disease Study 2016. The Lancet. 2017;390(10100):1260-1344.

21. Ministry of Health Nepal, New ERA, ICF. Nepal Demographic and Health Survey 2011. Kathmandu, Nepal: Ministry of Health; 2012.

22. Ministry of Health Nepal, New ERA, ICF. Nepal Demographic and Health Survey 2016. Kathmandu, Nepal: Ministry of Health; 2017.

23. Kurmi O, Regmi PR, Pant PR. Implication of air pollution on health effects in Nepal: lessons from global research. Nepal J Epidemiol. 2016; 6(1):525.

24. The World Bank Data Catalogue. World Bank Group; 2017. Available from: https://data.worldbank.org/indicator/SP.DYN.LE00.IN? end=2015\&start=1989. Accessed November 27, 2017.

25. GBD Compare Data Visualization. IHME, University of Washington; 2017. Available from: http://vizhub.healthdata.org/gbd-compare. Accessed September 17, 2017.

26. Nepal C. National population and housing census 2011 (National Report). Kathmandu, Nepal: Central Burea of Statistics; 2012:266.

27. Gautam R, Neupane D, Karki A, Kallestrup P. Community-based management of COPD in Nepal. Lancet Respir Med. 2017;5(1):e6.

28. Chen C, Zeger S, Breysse P, et al. Estimating indoor PM2. 5 and CO concentrations in households in Southern Nepal: the Nepal cookstove intervention trials. PLoS One. 2016;11(7):e0157984.

29. Ranabhat CL, Kim CB, Kim CS, Jha N, Deepak K, Connel FA. Consequence of indoor air pollution in rural area of Nepal: a simplified measurement approach. Front Public Health. 2015;3:5. 
30. Aryal KK. Non communicable Diseases Risk Factors: STEPS Survey Nepal 2013. Kathmandu: Nepal Health Research Council (NHRC); 2014

31. Ng M, Freeman MK, Fleming TD, et al. Smoking prevalence and cigarette consumption in 187 countries, 1980-2012. JAMA. 2014;311(2): 183-192.

32. Yamasaki-Nakagawa M, Ozasa K, Yamada N, et al. Gender difference in delays to diagnosis and health care seeking behaviour in a rural area of Nepal. Int J Tuberc Lung Dis. 2001;5(1):24-31.

33. Miller MR, Jordan RE, Adab P. Gender differences in COPD: are women more susceptible to smoking effects than men? Thorax. 2011; 66(10):921-922.

34. Hopkinson NS, Polkey MI. Does physical inactivity cause chronic obstructive pulmonary disease? Clin Sci. 2010;118(9):565-572.
35. Forouzanfar MH, Afshin A, Alexander LT, et al. Global, regional, and national comparative risk assessment of 79 behavioural, environmental and occupational, and metabolic risks or clusters of risks, 1990-2015: a systematic analysis for the Global Burden of Disease Study 2015. Lancet. 2016;388(10053):1659-1724.

36. Stevens GA, Alkema L, Black RE, et al. Guidelines for accurate and transparent health estimates reporting: the GATHER statement. PLoS Med. 2016;13(6):e1002056.

International Journal of COPD

\section{Publish your work in this journal}

The International Journal of COPD is an international, peer-reviewed journal of therapeutics and pharmacology focusing on concise rapid reporting of clinical studies and reviews in COPD. Special focus is given to the pathophysiological processes underlying the disease, intervention programs, patient focused education, and self management protocols.

\section{Dovepress}

This journal is indexed on PubMed Central, MedLine and CAS. The manuscript management system is completely online and includes a very quick and fair peer-review system, which is all easy to use. Visit http://www.dovepress.com/testimonials.php to read real quotes from published authors.

Submit your manuscript here: http://www.dovepress.com/international-journal-of-chronic-obstructive-pulmonary-disease-journal 